\title{
The fixed point property for ordered sets
}

Received: 1 June 2012 / Accepted: 7 September 2012 / Published online: 3 October 2012

(C) The Author(s) 2012. This article is published with open access at Springerlink.com

\begin{abstract}
An ordered set $P$ has the fixed point property iff every order-preserving self-map of $P$ has a fixed point. This paper traces the chronological development of research on this property, including most recent developments and open questions.
\end{abstract}

Mathematics Subject Classification 06A06

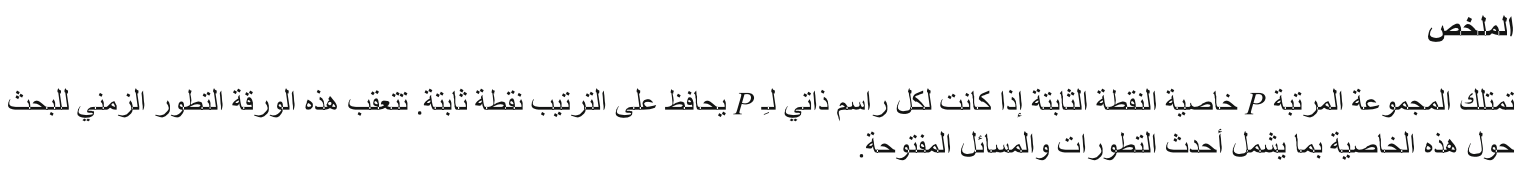

\section{Introduction}

An ordered set, or, a partially ordered set consists of a set $P$ and a reflexive, antisymmetric and transitive relation $\leq$, the order relation. Unless there is the possibility of confusing several order relations, we will refer to the underlying set $P$ as the ordered set. Subsets $S \subseteq P$ inherit the order relation from $P$ by restriction to $S$. Familiar examples of ordered sets include the number systems $\mathbb{N}, \mathbb{Z}, \mathbb{Q}$ and $\mathbb{R}$, as well as spaces of real valued functions, ordered by the pointwise order. Finite ordered sets are typically represented with a Hasse diagram as in Fig. 2. In a Hasse diagram, elements $x$ and $y$ satisfy the relation $x \leq y$ iff there is a path from $x$ to $y$ that may go through other elements of the set, but for which all segments are traversed in the upward direction.

The homomorphisms between ordered sets $P$ and $Q$ are the order-preserving functions, that is, functions $f: P \rightarrow Q$ so that $x \leq y$ (in $P$ ) implies $f(x) \leq f(y)$ (in $Q$ ). We will say that an ordered set $P$ has the fixed point property iff every order-preserving self-map $f: P \rightarrow P$ has a fixed point $x=f(x)$.

This survey presents the development of the fixed point property as a research topic in a by-and-large chronological fashion. Topics are presented in the order in which the first results occurred in the literature and they are discussed as fully as possible (regardless of chronology) before proceeding to the next topic. Readers who are interested in presentations from a different point-of-view could consider [44] or [46]. Definitions are presented when they are first needed. Finally, we will focus mainly on finite ordered sets and request the reader's patience when infinite arguments are unavoidable.

\section{B. S. W. Schröder $(\varangle)$}

Program of Mathematics and Statistics,

Louisiana Tech University, P.O. Box 10348, Ruston, LA 71272, USA

E-mail: schroder@coes.latech.edu 


\section{Knaster's proof (1928)}

The earliest fixed point result for ordered sets that the author is aware of occurs in Knaster's 1928 proof of the Bernstein-Cantor-Schröder ${ }^{1}$ Theorem from set theory in [25]. The Bernstein-Cantor-Schröder Theorem guarantees that when a set $A$ is "smaller than or of equal size as" the set $B$ (meaning there is an injective function from $A$ to $B$ ) and "greater than or of equal size as" the set $B$ (meaning there is an injective function from $B$ to $A$ ), then $A$ and $B$ have in fact "the same size" (meaning there is a bijective function between them).

Theorem 2.1 (The Bernstein-Cantor-Schröder Theorem) Let $A$ and $B$ be sets such that there is an injective function $f: A \rightarrow B$ and an injective function $g: B \rightarrow A$. Then there is a bijective function $h: A \rightarrow B$.

Proof (Inspired by [25]) Define a function $F: \mathcal{P}(A) \rightarrow \mathcal{P}(A)$ on the power set $\mathcal{P}(A)$ of $A$ by $F(C):=$ $A \backslash g[B \backslash f[C]]$. This function is order-preserving, because, if $C \subseteq D$, then $f[C] \subseteq f[D]$, which implies $B \backslash f[C] \supseteq B \backslash f[D]$, from which follows $g[B \backslash f[C]] \supseteq g[B \backslash f[D]]$, and finally

$$
F(C)=A \backslash g[B \backslash f[C]] \subseteq A \backslash g[B \backslash f[D]]=F(D) .
$$

The fixed point argument at the heart of this proof is now the following. Because $\emptyset \subseteq F(\emptyset)$, the set $\mathcal{U}:=\{S \subseteq A: S \subseteq F(S)\}$ is not empty. Let $E:=\bigcup\{S \subseteq A: S \subseteq F(S)\}$. Then, for all $S \subseteq A$ with $S \subseteq F(S)$, we have $S \subseteq E$, which means $S \subseteq F(S) \subseteq F(E)$. Hence the union of all sets $S \subseteq A$ with $S \subseteq F(S)$ must be contained in $F(E)$, that is, $E \subseteq F(E)$. It follows that $F(E) \subseteq F(F(E))$, which means $F(E) \in\{S \subseteq A: S \subseteq F(S)\}$, from which we conclude $F(E) \subseteq \bigcup\{S \subseteq A: S \subseteq F(S)\}=E$. This proves $E=F(E)$, that is, $E$ is a fixed point of $F$. (End of the fixed point argument.)

Now define $h: A \rightarrow B$ by

$$
h(x):= \begin{cases}f(x) ; & \text { if } x \in E, \\ g^{-1}(x) ; & \text { if } x \in A \backslash E\end{cases}
$$

The equation $E=F(E)=A \backslash g[B \backslash f[E]]$ implies $A \backslash E=g[B \backslash f[E]]$, which means that the function $h$ is totally defined. To see that $h$ is injective, let $x$ and $y$ be two distinct elements of $A$. Because $f$ and $g^{-1}$ are both injective, the only case we must consider is $x \in E$ and $y \in A \backslash E$. The containment $y \in A \backslash E=g[B \backslash f[E]]$ means there is a $z \in B \backslash f[E]$ such that $y=g(z)$. This in turn implies $h(y)=g^{-1}(y)=z \notin f[E]$ and since $h(x)=f(x) \in f[E]$, we have $h(x) \neq h(y)$.

To show that $h$ is surjective, let $b \in B$. If $b \in f[E]$, there is nothing to prove. If $b \in B \backslash f[E]$, then $g(b) \in g[B \backslash f[E]]=A \backslash E$. But then $b=g^{-1}(g(b))=h(g(b))$.

Note that the fixed point argument in the proof above only hinges on the following properties.

1. The power set $\mathcal{P}(A)$ is ordered by set containment $\subseteq$.

2. The power set $\mathcal{P}(A)$ has a smallest element. (Clearly, for $P$ an ordered set and $S \subseteq P$, we say $s \in S$ is the smallest element of $S$ iff, for all $x \in S$, we have $s \leq x$.) This assures that the set $\mathcal{U}$ is not empty.

3. Arbitrary unions can be formed. This assures that the union of $\mathcal{U}$ can be formed.

4. The function $F$ is order-preserving. This assures that containments are preserved the right way.

The order-theoretical analogue of a union is the supremum, defined below.

Definition 2.2 Let $P$ be an ordered set and let $A \subseteq P$. Then

1. The point $u$ is called an upper bound of $A$ iff, for all $a \in A$, we have $a \leq u$. The point $s$ is called the lowest upper bound or supremum of $A$, denoted $\bigvee A$, or, for $A=\{a, b\}, a \vee b$, iff $s$ is an upper bound of $A$ and for all upper bounds $u$ of $A$ we have $s \leq u$.

2. The point $l$ is called a lower bound of $A$ iff, for all $a \in A$, we have that $l \leq a$. The point $i$ is called the greatest lower bound or infimum of $A$, denoted $\bigwedge A$, or, for $A=\{a, b\}, a \wedge b$, iff $i$ is a lower bound of $A$ and for all lower bounds $l$ of $A$ we have $l \leq i$.

Ordered sets in which finite suprema and infima exist are the subject of lattice theory.

1 This theorem is often called the Cantor-Bernstein Theorem or the Schröder-Bernstein Theorem. Interestingly, Cantor only attempted to prove it, but did not succeed. The German mathematician Schröder (not a relative of the author) stated the theorem earlier, but his proof was flawed, too. It was Cantor's student Bernstein who finally supplied a correct proof in his dissertation. Let's hope we get it right here. 
Definition 2.3 Let $L$ be an ordered set. Then $L$ is called a lattice iff any two elements of $L$ have a supremum and an infimum. $L$ is called a complete lattice iff any subset of $L$ has a supremum and an infimum.

Note that a complete lattice $L$ has a smallest element: Every element $x$ of $L$ is an upper bound of the empty set, because $x$ is vacuously greater than or equal to every element of the empty set. Therefore the supremum of the empty set, which is guaranteed to exist by definition, will be less than or equal to every element of $L$. We denote the smallest element of $L$ by 0 .

\section{Tarski and Davis (1955), Abian and Brown (1961)}

Now, if $L$ is a complete lattice and $f: L \rightarrow L$ is an order-preserving function, then the fixed point argument from the proof of the Cantor-Schröder-Bernstein theorem translates verbatim: Because $0 \leq f(0)$, the set $\mathcal{U}:=\{x \in L: x \leq f(x)\}$ is not empty. Let $y:=\bigvee\{x \in L: x \leq f(x)\}$. Then, for all $x \in L$ with $x \leq f(x)$, we have $x \leq y$, which means $x \leq f(x) \leq f(y)$. Hence the supremum of all elements $x \in L$ with $x \leq f(x)$ must be below $f(y)$, that is $y \leq f(y)$. It follows that $f(y) \leq f(f(y))$, which means $f(y) \in\{x \in L: x \leq f(x)\}$, from which we conclude $f(y) \leq \bigvee\{x \in L: x \leq f(x)\}=y$. This proves that $y=f(y)$, that is, $y$ is a fixed point of $f$.

The above proves one direction of the theorem by Tarski and Davis that characterizes the fixed point property for lattices.

Theorem 3.1 (The Tarski-Davis theorem, see $[9,49])^{2}$ A lattice L has the fixed point property iff L is a complete lattice. In this situation, for each order-preserving map $f: L \rightarrow L$, the set $\operatorname{Fix}(f):=\{x \in P: f(x)=x\}$ is a complete lattice.

Proof The direction " $\Leftarrow$ " (published in [49]) has already been shown.

For the direction " $\Rightarrow$ " (published in [9]), let $L$ be a lattice with the fixed point property and suppose, for a contradiction, that $L$ is not complete. For this part of the proof, we need a few more definitions.

Definition 3.2 An ordered set $C$ is called a chain (or a totally ordered set or a linearly ordered set) iff for all $p, q \in C$ we have $p \leq q$ or $p \geq q$, which we will also denote by $p \sim q$.

Definition 3.3 An ordered set $W$ is called well-ordered iff each non-empty subset $A \subseteq W$ has a smallest element. (If we disregard the substantial set theoretical subtleties, then well-ordered sets can be visualized as copies of the natural numbers that are stacked on top of each other.)

Without loss of generality, assume that there is a non-empty subset $B \subseteq L$ that does not have a supremum. Because $L$ is a lattice, this set $B$ is infinite. Let $\beta$ be the smallest ordinal number of the same cardinality as $B .^{3}$ Index $B$ with $\beta$ as $B=\left\{b_{\alpha}: \alpha<\beta\right\}$. Inductively, for $\gamma \leq \beta$ consider the supremum $c_{\gamma}:=\bigvee\left\{b_{\alpha}: \alpha<\gamma\right\}$, if it exists. The supremum $c_{\beta}$ does not exist, because it would be the supremum of $B$.

Let $\gamma_{0}$ be the smallest $\gamma \leq \beta$ so that the supremum $c_{\gamma}$ does not exist. Then $C:=\left\{c_{\alpha}: \alpha<\gamma_{0}\right\}$ is a well-ordered chain that does not have a supremum in $L$ : Indeed, if $C$ had a supremum $s$, then $s$ would be an upper bound of $\left\{b_{\alpha}: \alpha<\gamma_{0}\right\}$. Moreover, if $u$ was an upper bound of $\left\{b_{\alpha}: \alpha<\gamma_{0}\right\}$, then $u$ would be an upper bound of $C$ and hence $u \geq s$. But this would mean that $s$ is the supremum of $\left\{b_{\alpha}: \alpha<\gamma_{0}\right\}$, a contradiction.

Because $C$ does not have a supremum, for every upper bound $u$ of $C$ there is another upper bound $u^{\prime}$ of $C$ so that $u^{\prime}<u$. Let $U$ be the set of upper bounds of $C$, let $\delta$ be the smallest ordinal number of the same cardinality as $U$ and index $U$ with $\delta$ as $U=\left\{u_{\alpha}: \alpha<\delta\right\}$. Let $d_{0} \in U$ be arbitrary. For each ordinal $\alpha$ that satisfies $0<\alpha<\delta$ and that has an immediate predecessor $\alpha^{-}$, define $d_{\alpha}:=d_{\alpha^{-}} \wedge u_{\alpha}$. For each limit ordinal $\lambda<\delta$ so that there is an element $x \in U$ that is below all $d_{\alpha}$ with $\alpha<\lambda$, choose $d_{\lambda}$ to be such an element. ${ }^{4}$ This process terminates with a dually well-ordered chain $^{5} D=\left\{d_{\alpha}: \alpha<\mu\right\}$, where $\mu$ is some limit ordinal, so that there is no element $x \in L$ that satisfies $c_{\alpha} \leq x$ for all $\alpha<\gamma_{0}$ and $x \leq d_{\alpha}$ for all $\alpha<\mu$ (see Fig. 1).

\footnotetext{
2 Although both papers were published in 1955, according to footnotes in Tarski's paper, Tarski proved his part of the result in 1939. Supposedly, he decided to publish it once Davis, who was his student, proved the converse and the papers appeared back-to-back in the same journal.

${ }^{3}$ For our purposes, it will be enough to think of ordinal numbers as certain "canonical well-ordered sets." Delving into the details of set theory reveals nothing more than that that's what they are.

${ }^{4}$ Readers versed in set theory will realize that we are skipping technical details here, not least of which is the fact that we need the Axiom of Choice. Because we will mainly focus on finite sets, this excursion into the infinite is kept as concise as possible.

5 In "dual" notions, all inequalities from the original definition are reversed, so this is a chain in which every non-empty subset has a largest element.
} 


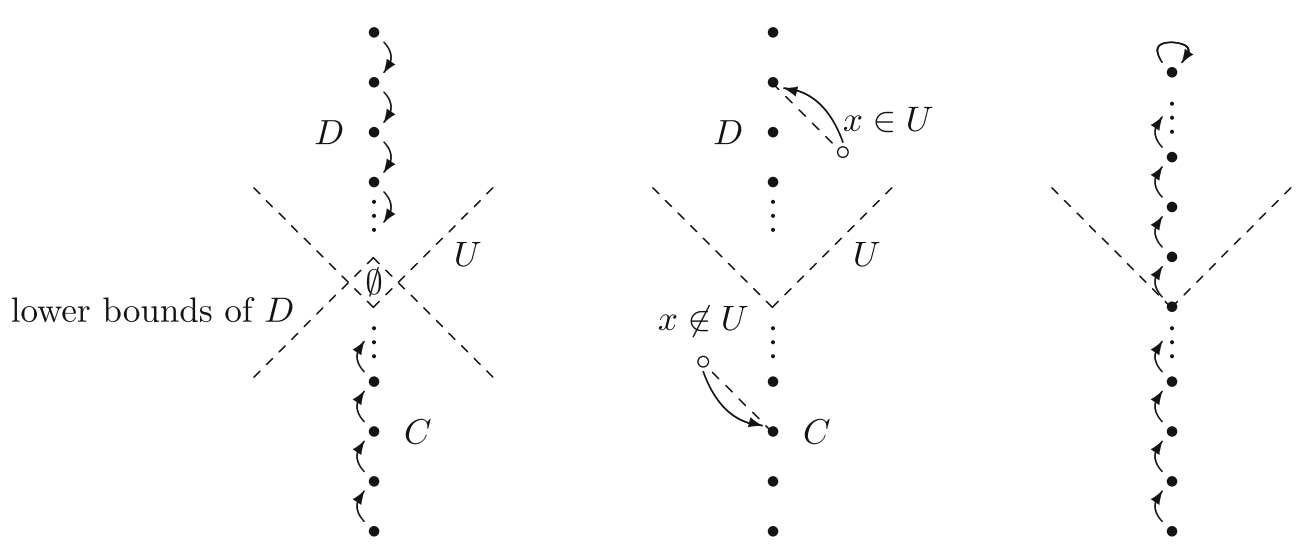

Fig. 1 The set $C \cup D$ and its fixed point free self-map from the proof of Davis' result (left), the function that maps $L$ to $C \cup D$ (center) and a visualization of "the usual upward iteration" (right)

Note that the union $C \cup D$ does not have the fixed point property. Indeed, for any ordinal number $\alpha$, let $\alpha^{+}$ denote the successor of $\alpha$. If we map every $c_{\alpha}$ to $c_{\alpha^{+}}$(which is the next element above $c_{\alpha}$, see left image in Fig. 1) and if we map every element $d_{\alpha}$ to $d_{\alpha^{+}}$(which, because $D$ is "upside down," is the next element below $d_{\alpha}$, see left image in Fig. 1), then the resulting function is order-preserving and does not have a fixed point. So we will be done with the proof if we can reduce focus from $L$ to $C \cup D$.

For every $x \in L$, there are two possibilities: Either $x$ is an upper bound of $C$ or it is not. If $x$ is not an upper bound of $C$, and there is no element of $C$ that is below $x$, map $x$ to the smallest element of $C$. If $x$ is not an upper bound of $C$, and an element of $C$ is below $x$, map $x$ to the largest element of $C$ that is below $x$. If $x$ is an upper bound of $C$, then $x$ is not a lower bound of $D$ and we map $x$ to the infimum in $D$ of the set of all elements of $D$ that are greater than or equal to $x$ (see center image in Fig. 1). It is easily checked that this function is an order-preserving function from $L$ to $C \cup D$ that fixes every element of $C \cup D$. Therefore the composition of this function with the function from the preceding paragraph is a fixed-point-free order-preserving self-map of $L$, contradicting the assumption that $L$ has the fixed point property.

We now return the proof from the argument in [9] back to arguments in [49], namely, the claim that Fix $(f)$ is a complete lattice. To prove this part, we first present the next "big" fixed point result in the literature, the Abian-Brown Theorem from 1961. Consider an order-preserving map $f: P \rightarrow P$ and a point $x$ so that $x \leq f(x)$. Then $f(x) \leq f(f(x)), f(f(x)) \leq f(f(f(x)))$ and, inductively, $f^{n}(x) \leq f^{n+1}(x) .{ }^{6}$ In a finite set, there will be an $n$ so that $f^{n}(x)=f^{n+1}(x)=f\left(f^{n}(x)\right)$, so the existence of a point $x \leq f(x)$ guarantees existence of a fixed point in finite sets. In infinite sets, the set $\left\{f^{n}(x): n \in \mathbb{N}\right\}$ can be infinite. Similar to the argument that was sketched for the construction of $D$ above, but in the upwards direction, we need a way to "get past the limit ordinal." To do this, we need suprema of non-empty chains. (And because the initial inequality could also have been $x \geq f(x)$, we also demand the existence of infima.)

Definition 3.4 Let $P$ be an ordered set. Then $P$ is called chain-complete iff each non-empty subchain $C \subseteq P$ has a supremum and an infimum.

In a chain-complete set, the set $\left\{f^{n}(x): n \in \mathbb{N}\right\}$ has a supremum $s$. Moreover, for all $n \in \mathbb{N}$, the inequality $s \geq f^{n}(x)$ implies $f(s) \geq f^{n+1}(x) \geq f^{n}(x)$, so that $f(s) \geq s$. $^{7}$ Hence, we can continue this process. Here is where, similar to the construction of $D$ above, the set theoretical technicalities must be carefully observed to obtain a correct proof. However, the simple truth is that this process continues in a transfinite fashion, pushing past limit ordinals by taking suprema (see right image in Fig. 1), until we reach a fixed point $p$. Because the image of every fixed point above $x$ must be above the whole transfinite chain that we constructed to obtain the fixed point $p$, the fixed point $p$ is in fact the smallest fixed point above $x$. We have thus sketched the proof of the following result.

Theorem 3.5 (The Abian-Brown Theorem, see [3]) Let $P$ be a chain-complete ordered set and let $f: P \rightarrow P$ be order-preserving. If there is an $x \in P$ with $x \leq f(x)$, then $f$ has a smallest fixed point above $x$.

${ }^{6}$ Note how the core idea of applying $f$ to $x \leq f(x)$ was already used in Knaster's argument.

7 Again, we use an argument that is similar to the one in the fixed point argument for the Bernstein-Cantor-Schröder Theorem. 

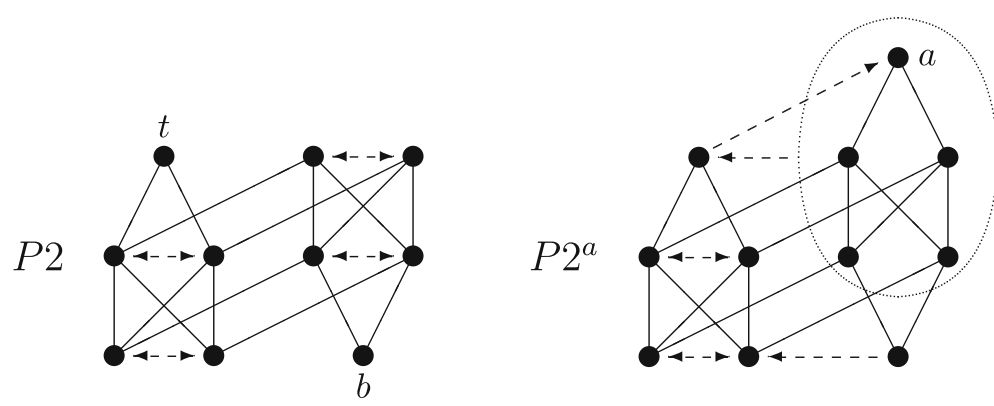

Fig. 2 The set $P 2$ (left) has the fixed point property and a self-map whose set of fixed points is disconnected. This self-map fixes the points $t$ and $b$ and it interchanges the other points as indicated by the arrows. The set $P 2^{a}$ on the right side and the indicated fixed point free order-preserving map will be discussed in Sect. 6

Similar to Tarski's result, though apparently without explicit statements in the literature, the Abian-Brown Theorem was supposedly known before the publication of [3], but [3] is the first paper that explicitly records this result. The technical details can be set up in such a way that the Axiom of Choice is not needed (see [2]). Because the result and the argument are so natural, the Abian-Brown Theorem is being quoted as well as rediscovered by researchers to this day. It is most commonly used in discrete mathematics, say, in directed sets (see [8]), but Heikkilä also uses Abian-Brown type results in analysis (see [17] for an example and [18] for a monograph).

Now, to prove that $\operatorname{Fix}(f)$ is a complete lattice, let $A \subseteq \operatorname{Fix}(f)$ be not empty. Let $x:=\bigvee A$ be the supremum of $A$ in $L$. Then every fixed point of $f$ that is above $A$ is also above $x$. Moreover

$$
f(x)=f[\bigvee A] \geq \bigvee f[A]=\bigvee A=x .
$$

By the Abian-Brown Theorem, there is a smallest fixed point $a$ above $x$ and $a$ must be the supremum of $A$ in Fix $(f)$. Dually, $A$ has an infimum, which establishes that Fix $(f)$ is a complete lattice.

The arguments so far have already exhibited the most common tools for working with the fixed point property of ordered sets.

- "The usual (Abian-Brown) iteration" produces fixed points from points $x \leq f(x)$ or $x \geq f(x)$.

- The retraction of ordered sets to smaller ones, such as the function that mapped $L$ to $C \cup D$ in Davis' argument, is used to establish the fixed point property for smaller sets or to disprove it for larger sets.

We will formalize retractions in the next section and we will stay with finite or chain-complete ordered sets until Sect. 8.1. One feature of the Tarski-Davis Theorem that is rarely duplicated is that the theorem not just establishes that the set of fixed points is non-empty, but that it also establishes a property of the set of fixed points. This leads us to a first open problem.

Open Question 3.6 Given a family of ordered sets with the fixed point property, what additional properties (besides non-emptyness) do the sets of fixed points have?

This question is deliberately vague, because it is not clear at all what properties of the fixed point set should be guaranteed under what circumstances. For example, the ordered set $P 2$ in Fig. 2, which first appeared in [40], has the fixed point property, but it also has a function $f$ so that $\operatorname{Fix}(f)$ is disconnected.

\section{Rival's dismantlability (1976) and retractions}

The next big milestone in the fixed point theory for ordered sets is Rival's 1976 paper [35]. It uses retractions in the way Davis used them (an ordered set with the fixed point property cannot be retracted onto an ordered set that does not have the fixed point property), but, for the first time, it also uses the fixed point property of the retract to establish the fixed point property for the surrounding ordered set.

Definition 4.1 Let $P$ be an ordered set. Then an order-preserving map $r: P \rightarrow P$ is called a retraction iff $r^{2}=r$ (that is, iff $r$ is idempotent). We will say that $R \subseteq P$ is a retract of $P$ iff there is a retraction $r: P \rightarrow P$ with $r[P]=R$. 


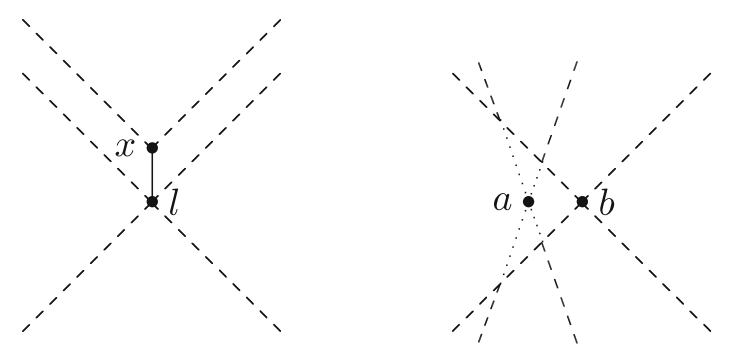

Fig. 3 Left An irreducible point $x$ with a unique lower cover $l$. Right A point $a$ that is retractable to the point $b$. Dashed lines indicate the sets of upper and lower bounds of the point. Dotted lines indicate an empty set

Theorem 4.2 Let $P$ be an ordered set with the fixed point property and let $r: P \rightarrow P$ be a retraction. Then $r[P]$ has the fixed point property.

Proof Let $f: r[P] \rightarrow r[P]$ be order-preserving. Then $f \circ r: P \rightarrow P$ is order-preserving, too, and hence it has a fixed point $x=f(r(x))$. But then $x \in f[P] \subseteq r[P]$ and hence $x=r(x)$, which means that $x=f(r(x))=f(x)$ is a fixed point of $f$.

Rival now considered irreducible points, which play an important role in lattice theory, in arbitrary ordered sets.

Definition 4.3 Let $P$ be an ordered set and let $x, y \in P$. If $x<y$ and there is no $z \in P$ so that $x<z<y$, then $y$ is called an upper cover of $x$ and $x$ is called a lower cover of $y$. In a finite ordered set, a point is called irreducible (see Fig. 3, left) iff it has exactly one upper cover or exactly one lower cover.

Let $x \in P$ be irreducible and assume without loss of generality that $x$ has a unique lower cover $l$. Then the function that fixes all points in $P \backslash\{x\}$ and which maps $x$ to $l$ is a retraction.

Theorem 4.4 (See [35], Proposition 1) Let $P$ be an ordered set and let $x \in P$ be irreducible. Then $P$ has the fixed point property iff $P \backslash\{x\}$ has the fixed point property.

Proof The direction " $\Rightarrow$ " follows from Theorem 4.2, so we only need to consider the direction " $\Leftarrow$." Without loss of generality, let $l$ be the unique lower cover of $x$ and let $f: P \rightarrow P$ be order-preserving. Let $r: P \rightarrow P \backslash\{x\}$ be the retraction that maps $x$ to $l$ and fixes all other points. Then $\left.r \circ f\right|_{r[P]}$ has a fixed point $z=r(f(z))$. If this fixed point is not $l$, then it is a fixed point of $f$. In case $z=l$, if $f(l)=l$, then $f$ has a fixed point. This leaves the case that $z=l$ and $f(l) \neq l$, which, because $r(f(l))=l$, means that $f(l)=x>l$.

Let $u \geq x$. Then $u \geq l$ and hence $f(u) \geq f(l)=x$. Hence $f$ maps the set $\{u \in P: u \geq x\}$ to itself. But this set is a retract of $P$ via the function that fixes all points in $\{u \in P: u \geq x\}$ and maps all other points to $x$. Hence the set $\{u \in P: u \geq x\}$ has the fixed point property and $f$ has a fixed point in $\{u \in P: u \geq x\}$, which completes the proof.

Theorem 4.4 suggests the following definition.

Definition 4.5 A finite ordered set $P$ with $n$ elements is called dismantlable by irreducibles iff there is an enumeration $p_{1}, \ldots, p_{n}$ of the elements of $P$ so that, for all $i=1, \ldots, n-1$, the point $p_{i}$ is irreducible in $P \backslash\left\{p_{1}, \ldots, p_{i-1}\right\}$.

Clearly, if $P$ is dismantlable by irreducibles, then, by Theorem 4.4, $P$ has the fixed point property. This idea of dismantlability by irreducibles is used in [35] to characterize the fixed point property for ordered sets of height 1 .

Definition 4.6 The length of a finite chain $C$ is $|C|-1$, so it is the number of steps we can go up in the chain, which is one less than the number of levels. The height of an ordered set is the length of the longest chain in the ordered set.

Definition 4.7 Let $P$ be an ordered set. An element $m \in P$ is called maximal iff for all $p \in P$ with $p \sim m$ we have $p \leq m$. Minimal elements are defined dually.

Hence, an ordered set of height 1 consists of two levels, a level of maximal elements and a level of minimal elements. 

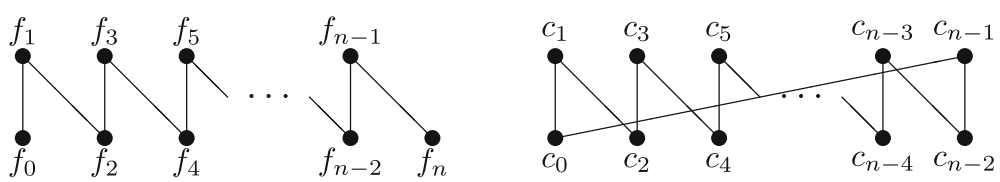

Fig. 4 Pictures of a fence (left) and a crown (right)

Definition 4.8 Let $P$ be an ordered set. An $(n+1)$-fence (see Fig. 4) is an ordered set $F=\left\{f_{0}, \ldots, f_{n}\right\}$ such that $f_{0}<f_{1}>f_{2}<\cdots f_{n}$ or $f_{0}>f_{1}<f_{2}>\cdots f_{n}$, and such that these are all strict comparabilities between the points. The length of the fence is $n$. The points $f_{0}$ and $f_{n}$ are called the endpoints of the fence.

The distance $d(a, b)$ between two points $a, b \in P$ is the length of the shortest fence from $a$ to $b$, if such a fence exists. Otherwise, we set the distance between $a$ and $b$ to infinity. We call an ordered set connected iff the distance between any two of its points is finite.

Disconnected ordered sets do not have the fixed point property, because, for $a, b$ with $d(a, b)=\infty$, we could map all points whose distance to $a$ is finite to $b$ and all other points to $a$. Hence we will focus on connected ordered sets.

Definition 4.9 Let $n \in \mathbb{N}$ be even and $\geq 4$. An $n$-crown (see Fig. 4) is an ordered set $C_{n}$ with point set $\left\{c_{0}, \ldots, c_{n-1}\right\}$ such that $c_{0}<c_{1}>c_{2}<c_{3}>\cdots>c_{n-2}<c_{n-1}>c_{0}$ are the only strict comparabilities.

Mapping $c_{i}$ to $c_{i+2}(\bmod n)$ shows that crowns do not have the fixed point property. There are two kinds of ordered sets of height 1 : Those that contain a crown and those that do not.

Lemma 4.10 Let $P$ be an ordered set of height 1 and let $C=\left\{c_{0}, \ldots, c_{n-1}\right\}$ be a crown of minimal size in $P$. Then $C$ is a retract of $P$.

Proof Assume without loss of generality that $c_{0}$ is minimal in $P$. Let $P^{\prime}$ be the ordered set obtained from $P$ by erasing the comparability $c_{0}<c_{n-1}$. With $d_{P^{\prime}}$ denoting the distance in $P^{\prime}$, we define

$$
r(x):= \begin{cases}c_{n-1} ; & \text { if } d_{P^{\prime}}\left(x, c_{0}\right) \geq n, \\ c_{i} ; & \text { if } d_{P^{\prime}}\left(x, c_{0}\right)=i<n .\end{cases}
$$

Because $C$ is a crown of smallest possible size in $P$, we have $d_{P^{\prime}}\left(c_{i}, c_{0}\right)=i$ for all $i$. Hence the map $r$ is idempotent. Now let $x<y$ (in $P$ ). Because $r$ clearly preserves the comparability $c_{0}<c_{n-1}$, we can assume $x \neq c_{0}$ or $y \neq c_{n-1}$. Then $d_{P^{\prime}}\left(x, c_{0}\right)$ is even and $d_{P^{\prime}}\left(y, c_{0}\right) \in\left\{d_{P^{\prime}}\left(x, c_{0}\right)-1, d_{P^{\prime}}\left(x, c_{0}\right)+1\right\}$. Thus if $d_{P^{\prime}}\left(x, c_{0}\right) \geq n$, then $r(x)=r(y)=c_{n-1}$, while if $d_{P^{\prime}}\left(x, c_{0}\right)<n$, then $r(x)$ is a minimal element of $C$ and $r(y)$ is one of its maximal upper covers in $C$. Thus $r$ is order-preserving and we are done.

Lemma 4.11 Let $P$ be a finite connected ordered set of height 1 that does not contain any crowns. Then $P$ must contain an irreducible point.

Proof We prove the contrapositive. Suppose $P$ has no irreducible points and let $p_{0} \in P$ be minimal. Let $p_{1}$ be an upper cover of $p_{0}$. Then $p_{1}$ is maximal and has at least two lower covers. Thus $p_{1}$ has a lower cover $p_{2} \neq p_{0}$. Suppose mutually distinct $p_{0}, \ldots, p_{n}$ such that $\left\{p_{0}, \ldots, p_{n}\right\}$ is a fence have been constructed already and, without loss of generality, assume that $p_{n}$ is minimal. Then $p_{n}$ has at least two upper covers, so $p_{n}$ has a maximal upper cover $p_{n+1} \neq p_{n-1}$.

Because $P$ is finite, this construction must ultimately produce a $p_{n+1}$ that is comparable to an earlier $p_{j}$ with $j<n$. Let $j_{m}$ be the largest $j<n$ such that $p_{n+1}$ is comparable to $p_{j}$. Then $\left\{p_{j_{m}}, \ldots, p_{n+1}\right\}$ is a crown.

Theorem 4.12 (See [32,35]) Let $P$ be a connected finite ordered set of height 1 . Then the following are equivalent.

1. $P$ has the fixed point property.

2. P contains no crowns.

3. $P$ is dismantlable by irreducibles.

Proof " $1 \Rightarrow 2$ " follows directly from Lemma 4.10 , " $2 \Rightarrow 3$ " follows from Lemma 4.11 and " $3 \Rightarrow 1$ " follows from Theorem 4.4 by induction. 
Theorems 3.1 and 4.12 are "best possible" fixed point theorems, as they characterize the fixed point property for natural classes of ordered sets with a natural criterion. In 1987, Fofanova and Rutkowski proved another such theorem.

Theorem 4.13 (See [15]) A finite ordered set of width 2 (in any set of three distinct elements, at least two are comparable) has the fixed point property iff it is dismantlable by irreducibles.

They also characterized the fixed point property for chain-complete infinite ordered sets of width 2. Dismantlability by irreducibles is also the key to characterizing the fixed point property for interval orders, as a finite ordered set with an interval order has the fixed point property iff it is dismantlable by irreducibles (see Proposition 8.2.2 in [46]).

The idea of removing a single point from the set can be extended as follows.

Definition 4.14 Let $P$ be an ordered set. A point $a \in P$ is called retractable (to $b \in P \backslash\{a\}$ ) iff for all $x \in P$, $x>a$ implies $x \geq b$ and $x<a$ implies $x \leq b$ (see Fig. 3, right).

The function that maps a retractable point $a$ to the point $b$ that it is retractable to, and which leaves all other points fixed, is a retraction from $P$ to $P \backslash\{a\}$. Moreover, irreducible points are retractable, and a retractable point $a$ is irreducible iff it is comparable to the point $b$ that it is retractable to.

Theorem 4.15 (See [43], Theorem 3.3) Let $P$ be an ordered set and let $a \in P$ be retractable to $b \in P$. Then $P$ has the fixed point property iff

1. $P \backslash\{a\}$ has the fixed point property, and

2. One of $\{p \in P: p>a\}$ and $\{p \in P: p<a\}$ has the fixed point property.

Proof " $\Rightarrow$." Let $P$ have the fixed point property. Condition 1 is a consequence of Theorem 4.2. For part 2, suppose for a contradiction that neither $\{p \in P: p>a\}$ nor $\{p \in P: p<a\}$ has the fixed point property. Let $g:\{p \in P: p>a\} \rightarrow\{p \in P: p>a\}$ and $h:\{p \in P: p<a\} \rightarrow\{p \in P: p<a\}$ be order-preserving maps without fixed points. Define

$$
f(q):= \begin{cases}g(q) ; & \text { if } q>a \\ h(q) ; & \text { if } q<a \\ a ; & \text { if } q \text { is not comparable to a, } \\ b ; & \text { if } q=a\end{cases}
$$

Clearly $f: P \rightarrow P$ has no fixed point and it is not too hard to verify that $f$ is order-preserving. This is the desired contradiction.

" $\Leftarrow$." Let $f: P \rightarrow P$ be order-preserving. We will show that $f$ has a fixed point. Let $r: P \rightarrow P \backslash\{a\}$ be the retraction that maps $a$ to $b$. Then $\left.r \circ f\right|_{P \backslash\{a\}}: P \backslash\{a\} \rightarrow P \backslash\{a\}$ is order-preserving and thus it has a fixed point $q$. In case $q \neq b$ we have that $q=\left.r \circ f\right|_{P \backslash\{a\}}(q)=\left.f\right|_{P \backslash\{a\}}(q)=f(q)$ and we are done. In case $q=b$ we either have $f(b)=b$ or $f(b)=a$. In the first case, we are done. In the second case, assume without loss of generality that $\{p \in P: p>a\}$ has the fixed point property. Then $\{p \in P: p>a\} \neq \emptyset$. Because $f(b)=a$, we have that $f[\{p \in P: p>a\}] \subseteq f[\{p \in P: p \geq b\}] \subseteq\{p \in P: p \geq a\}$. In case $f[\{p \in P: p>a\}] \subseteq\{p \in P: p>a\}$, the function $f$ has a fixed point in $\{p \in P: p>a\}$ and we are done. Otherwise there is a $q \in\{p \in P: p>a\}$ such that $f(q)=a<q$. If $f(a)=a$, we are done. This leaves the case that $d:=f(a)<a$. In this case, $f$ maps $\{p \in P: p \leq d\}$ to itself. Yet $\{p \in P: p \leq d\}$ is a retract of $P \backslash\{a\}$ via an argument similar to that in the last paragraph of the proof of Theorem 4.4. Thus $\{p \in P: p \leq d\}$ has the fixed point property, which implies that $f$ has a fixed point in $\{p \in P: p \leq d\}$.

Retractable points play a role for the fixed point property for ordered sets of dimension 2.

Definition 4.16 The dimension of an ordered set is the smallest number $n$ of total order relations $\leq_{i}$ so that $\leq=\bigcap_{i=1}^{n} \leq_{i}$. If we replace total orders with interval orders, we obtain the notion of interval dimension.

Fofanova, Rival and Rutkowski proved in [16] that every ordered set of dimension 2 has a retractable point. The same result can be proved for ordered sets of interval dimension 2, see Theorem 8.4.5 of [46].

Note that, when using Theorem 4.15 to check the fixed point property for ordered sets of (interval) dimension 2 , we no longer simply obtain new sets by removing one element after another in a linear fashion. Because Theorem 4.15 also requires one of $\{p \in P: p>a\}$ or $\{p \in P: p<a\}$ to have the fixed point property, at each stage the fixed point property must be verified for $P \backslash\{a\}$ and for one of $\{p \in P: p>a\}$ or $\{p \in P: p<a\}$, which is the same as verifying it for $\{p \in P: p \sim a\}$. 


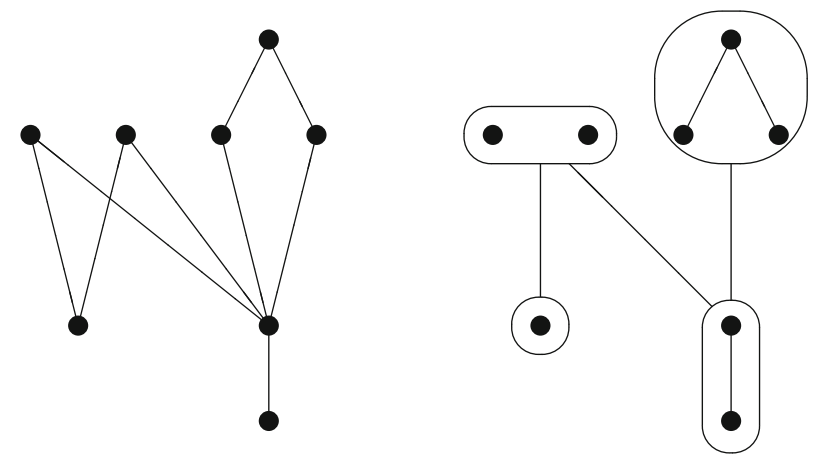

Fig. 5 In a lexicographic sum, elements of an ordered set (left) are contained in smaller sets (identified by bubbles on the right) that are "summed" through an index set (a 4-fence in this case)

Definition 4.17 A finite ordered set $P$ is called collapsible iff $P$ has a retractable point $x$ so that $P \backslash\{x\}$ and $\{p \in P: p \sim x\}$ are collapsible.

We know that ordered sets of (interval) dimension 2 are collapsible. Verification of the fixed point property for a collapsible ordered set requires generating a binary tree of subsets so that all leaves of the tree can be verified to have the fixed point property. We will revisit this observation in Sect. 9.

\section{Höft and Höft's work on lexicographic sums (1976)}

In the same year as Rival's seminal paper [35], Höft and Höft published the first in a sequence of papers on lexicographic sums. (We condense their results here into Theorem 5.4.) Essentially, a lexicographic sum is an ordered set $T$ in which each element has been replaced with another non-empty ordered set and, for any two elements $a<b$ that are comparable in $T$, the new ordered sets are placed so that the set that replaces $a$ is completely below the set that replaces $b$ (see Fig. 5).

Definition 5.1 Let $T$ be a non-empty ordered set considered as an index set. Let $\left\{P_{t}\right\}_{t \in T}$ be a family of pairwise disjoint non-empty ordered sets that are all disjoint from $T$. We define the lexicographic sum $L\left\{P_{t} \mid t \in T\right\}$ of the $P_{t}$ over $T$ to be the set $\bigcup_{t \in T} P_{t}$ ordered by $p_{1} \leq p_{2}$ iff

1. $p_{i} \in P_{t_{i}}, t_{1} \neq t_{2}$ and $t_{1}<t_{2}$, or

2. $p_{1}, p_{2} \in P_{t}$ and $p_{1} \leq P_{t} p_{2}$.

The $P_{t}$ will be called the pieces of the lexicographic sum and $T$ will be called the index set. For $p \in L\left\{P_{t} \mid t \in T\right\}$ define $I(p)$ to be the unique $t \in T$ such that $p \in P_{t}$. If $T$ is a chain $t_{1}<t_{2}<\cdots<t_{n}$, the lexicographic sum $L\left\{P_{t} \mid t \in T\right\}$ will also be denoted by $P_{t_{1}} \oplus P_{t_{2}} \oplus \cdots \oplus P_{t_{n}}$.

Lemma 5.2 Let $P:=L\left\{P_{t} \mid t \in T\right\}$ be a lexicographic sum. Then $P$ has a retract that is isomorphic to $T$.

Proof For each $t \in T$ choose an element $p_{t} \in P_{t}$. The map that maps each piece $P_{t}$ to the chosen element $p_{t}$ is a retraction of $P$ to an ordered set that is isomorphic to $T$.

So if a lexicographic sum has the fixed point property, then the index set must have the fixed point property, too. The challenge is now to determine which pieces must have the fixed point property so that the overall lexicographic sum has the fixed point property. The next lemma shows that, for index sets that are chains, there is no specific individual piece that must have the fixed point property.

Lemma 5.3 Let $P:=L\left\{P_{c} \mid c \in C\right\}$ be a chain-complete lexicographic sum with a chain $C$ as index set. Then $P$ has the fixed point property iff one piece $P_{c}$ has the fixed point property.

Proof If none of the pieces have the fixed point property, applying a fixed point free order-preserving self-map on each piece shows that $P$ does not have the fixed point property.

Now consider the case in which one $P_{c}$ has the fixed point property. Let $f: P \rightarrow P$ be order-preserving. If $f$ maps $P_{c}$ to itself, then $f$ has a fixed point in $P_{c}$. Otherwise, for some $p_{c} \in P_{c}$, we have $f\left(p_{c}\right) \sim p_{c}$ and hence, by the Abian-Brown Theorem, $f$ must have a fixed point.

Now that we know that the fixed point property for a lexicographic sum need not imply the fixed point property for any specific individual piece, the following result appears to be best possible. 
Theorem 5.4 (Compare with [22-24]) A chain-complete lexicographic sum $P=L\left\{P_{t} \mid t \in T\right\}$ has the fixed point property iff

1. $T$ has the fixed point property, and

2. For all $t_{0} \in T$, one of $P_{t_{0}}, L\left\{P_{t} \mid t<t_{0}\right\}$ and $L\left\{P_{t} \mid t>t_{0}\right\}$ has the fixed point property.

Proof First suppose $P$ has the fixed point property. By Lemma 5.2, part 1 must hold. For part 2, fix a point $p_{t_{0}} \in P_{t_{0}}$. The map that fixes all points that are comparable to some $p \in P_{t_{0}}$ and which maps all other points to $p_{t_{0}}$ is a retraction of $P$ onto $L\left\{P_{t} \mid t<t_{0}\right\} \oplus P_{t_{0}} \oplus L\left\{P_{t} \mid t>t_{0}\right\}$. Thus $L\left\{P_{t} \mid t<t_{0}\right\} \oplus P_{t_{0}} \oplus L\left\{P_{t} \mid t>t_{0}\right\}$ must have the fixed point property. By Lemma 5.3 this is the case iff one of its three pieces has the fixed point property. Thus 2 must hold.

Conversely, suppose $P=L\left\{P_{t} \mid t \in T\right\}$ satisfies conditions 1 and 2. Let $f: P \rightarrow P$ be an order-preserving map. Define $F: T \rightarrow \mathcal{P}(T) \backslash\{\emptyset\}$ by $F(t):=I\left[P_{t}\right]$. Then, for some $t \in T$, we must have $F(t)=\{t\}$ : Otherwise, for each $t \in T$, choose $g(t) \in F(t) \backslash\{t\}$. This map would be an order-preserving fixed point free selfmap of $T$, a contradiction to 1 . Find a $t_{0} \in T$ with $F\left(t_{0}\right)=\left\{t_{0}\right\}$. Then $f$ maps $L\left\{P_{t} \mid t<t_{0}\right\} \oplus P_{t_{0}} \oplus L\left\{P_{t} \mid t>t_{0}\right\}$ to itself. Because, by 2 , one of the three pieces must have the fixed point property, we conclude via Lemma 5.3 that $f$ has a fixed point.

\section{Baclawski, Björner and the fixed simplex property (1979)}

The next milestone is Baclawski and Björner's 1979 paper [5] that connects the fixed point property for ordered sets to the fixed point property for continuous functions in topology and to algebraic topology, in particular, to homology. The details of homology are beyond the scope of this paper, but they can be found in [44] and in [46]. Instead of these intricacies, we will describe the necessary translations and latest developments.

Definition 6.1 The comparability graph $C(P)$ of an ordered set $P$ has as its vertices the elements of $P$ and there is an edge between two distinct vertices $x$ and $y$ iff $x \sim y$.

Definition 6.2 Let $G=(V, E)$ be a graph. A function $f: V \rightarrow V$ will be called a simplicial endomorphism of $G$ iff for all $x \sim y$ in $G$ we have $f(x) \sim f(y)$ or $f(x)=f(y)$.

Simplicial endomorphisms are not homomorphisms or endomorphisms in the sense that is more commonly used in graph theory (also see Sect. 10). However, if $f: P \rightarrow P$ is order-preserving, then $f$ is also a simplicial endomorphism on the comparability graph $C(P)$.

The fixed point property for simplicial endomorphisms of graphs is a rather disappointing affair. Let $G$ be a graph that has at least one edge $x \sim y$. Then the function which maps $x$ to $y$ and all other vertices to $x$ is a simplicial endomorphism that has no fixed points. Rather than the fixed point property, for graphs and simplicial endomorphisms, the fixed clique property, which first appeared in the guise of a fixed edge property in [32], is more appropriate to use.

Definition 6.3 A clique in a graph is a set $C$ of vertices so that any two distinct elements of $C$ are adjacent. A graph $G$ has the fixed clique property iff, for every simplicial endomorphism of $G$, there is a clique $C$ so that $f[C]=C$.

Clearly, if the comparability graph $C(P)$ of a finite ordered set $P$ has the fixed clique property, then $P$ has the fixed point property: Indeed, if $f: P \rightarrow P$ is order-preserving, then $f$ is a simplicial endomorphism. Hence there is a clique $C$ with $f[C]=C$. The cliques of $C(P)$ are exactly the chains of $P$, so $f$ maps a chain of $P$ to itself, which implies existence of a fixed point via the Abian-Brown Theorem.

The converse, however, is not true. Consider once more the set $P 2$ in Fig. 2, which has the fixed point property. The $180^{\circ}$ rotation about the picture's center is a simplicial endomorphism of the comparability graph that does not have any fixed cliques.

Note also, that there is no Abian-Brown Theorem for the fixed clique property. In a 4-cycle $v_{1} \sim v_{2} \sim$ $v_{3} \sim v_{4} \sim v_{1}$, the function defined by $f\left(v_{i}\right)=v_{i+1}(\bmod 4)$ is a simplicial isomorphism so that $v_{i} \sim f\left(v_{i}\right)$ for all $i$ and yet it has no fixed clique.

Although the fixed clique property is only an intermediate stage here, and although attention to the fixed clique property has been sporadic at best, it has found applications recently in universal algebra (see [30]).

We now turn our attention to the objects that connect ordered sets and graphs to (algebraic) topology.

Definition 6.4 A simplicial complex on a set $V$ is a set $\Sigma \subseteq \mathcal{P}(V)$ so that, for all $\sigma \in \Sigma$, every subset of $\sigma$ is in $\Sigma$, too. The elements of $V$ are called the vertices of $\Sigma$ and the elements of $\Sigma$ are the simplices of $\Sigma$. 
Any graph can be viewed as a simplicial complex whose vertices are the vertices of the graph and whose simplices are the cliques of the graph. Hence, through the comparability graph, any ordered set has a simplicial complex associated with it, too.

Definition 6.5 Let $P$ be an ordered set. The chain complex $\Delta(P)$ of $P$ is the simplicial complex $\sigma$ that consists of all chains of $P$.

Definition 6.6 Let $\Sigma$ be a simplicial complex on the vertex set $V$. A function $f: V \rightarrow V$ is called a simplicial map iff for all $\sigma \in \Sigma$ we have that $f(\sigma) \in \Sigma$.

Clearly, for a graph, every simplicial endomorphism is a simplicial map on the corresponding simplicial complex and vice versa. ${ }^{8}$ Consequently, every order-preserving map of an ordered set is a simplicial map on the chain complex. However, the rotation of the set $P 2$ in Fig. 2 shows that not every simplicial map induces an order-preserving map.

Definition 6.7 Let $\Sigma$ be a simplicial complex on the set $V$ that has no infinite simplices. We say that $\Sigma$ has the fixed simplex property iff, for each simplicial map $f: V \rightarrow V$, there is a simplex $\sigma \in \Sigma \backslash\{\emptyset\}$ with $f(\sigma)=\sigma$.

Because, for graphs, simplicial endomorphisms and simplicial maps on the corresponding simplicial complex are the same thing, a graph $G$ has the fixed clique property iff the corresponding simplicial complex has the fixed simplex property. Hence, if the chain complex $\Delta(P)$ of an ordered set $P$ has the fixed simplex property, then $P$ has the fixed point property, but not conversely.

Simplicial maps now turn out to be continuous functions on the topological realization of the simplicial complex. (It goes without saying that not every continuous function is a simplicial map.) Therefore, we have the following.

Theorem 6.8 If the topological realization of the chain complex $\Delta(P)$ of a finite ordered set $P$ has the topological fixed point property (every continuous self-map has a fixed point), then $P$ has the fixed point property.

In particular, any sufficient condition for the topological fixed point property can be translated into a sufficient condition for the fixed point property for ordered sets. For example, we can compute the homology of the chain complex, and use algebraic topology to investigate the fixed point property.

Theorem 6.9 If the chain complex $\Delta(P)$ of a finite ordered set $P$ is acyclic (definition omitted), then the ordered set has the fixed point property.

The notion of acyclicity, for which we refer the reader to [44,46] or texts on algebraic topology, plays a big role in what is possibly the most well-known result from [5].

Definition 6.10 A lattice is called non-complemented iff for any two elements $x$ and $y$ we have that $x \vee y$ is not the largest element of the lattice or $x \wedge y$ is not the smallest element. A truncated lattice is obtained from a lattice by removing the largest and smallest elements.

Theorem 6.11 The chain complex of a finite truncated non-complemented lattice is acyclic. Hence finite truncated non-complemented lattices have the fixed point property.

This result has perplexed researchers on the fixed point property for ordered sets since its publication, because, until recently, every proof required the use of homology. The homology arguments typically used a theorem which said that, if $a \in P$ and $P \backslash\{a\}$ and $\{p \in P: p \sim a, p \neq a\}$ are both acyclic, then $P$ is acyclic, too. In the recent paper [4], Baclawski proves a similar result using combinatorial arguments and techniques similar to discrete Morse theory. Although the arguments are combinatorial, they will always remain connected to something more than a fixed point property and the removal of a single point: Consider the ordered set $P 2^{a}$ in Fig. 2, which does not have the fixed point property. The point $a$ is so that the set $\{p \in P: p \sim a, p \neq a\}=\{p \in P: p<a\}$ is dismantlable by irreducibles and it is known that these sets are acyclic. Removal of $a$ leads to the set $P 2$, which has the fixed point property, and yet $P 2^{a}$ does not have the fixed point property. Since all the above mentioned results are actually results about the fixed simplex property, we can also consider the following.

\footnotetext{
${ }^{8}$ Hence, although it is not canonical, the name "simplicial endomorphism."
} 
Open Question 6.12 Which results on the fixed point property have analogues for the fixed simplex property?

This question is non-trivial, because there is no Abian-Brown Theorem for the fixed simplex property. The author has proved an analogue of Theorem 4.15 for the fixed clique property. For the fixed simplex property, the analogue of condition 2 is not a necessary condition for the fixed simplex property anymore and the author has yet to complete the proof that the analogues of 1 and 2 are sufficient for the fixed simplex property (if this is indeed true; there may be further unexpected subtleties when we consider the fixed simplex property).

Question 6.12 also has implications for the fixed point property. The set of non-empty simplices, ordered by inclusion, is a truncated lattice and it can be shown that a finite simplicial complex has the fixed simplex property iff the set of non-empty simplices has the fixed point property (see Theorem 6.3.16 in [46]).

\section{Rutkowski's small sets (1989)}

Encouraged [1] by results for special classes of finite ordered sets such as Theorem 4.12, as well as by Theorem 3.1, which is quite removed from finite ordered sets, Rival asked if it was possible to characterize the fixed point property for finite ordered sets. The results in Sect. 4 show that there are several classes of ordered sets for which a characterization is possible. On the other hand, Sects. 5 and 6 indicate that the general situation is harder.

With dismantlability being well-understood, in 1989, Rutkowski produced, up to isomorphism and duality, all non-dismantlable ordered sets of size 10 or smaller that have the fixed point property: There are no such sets of size $\leq 8$, one such set of size 9 and 9 such sets of size 10. To this day, the examples on this list are valuable as test cases as well as inspiration for theorems.

The first ordered set on Rutkowski's list inspired Theorem 4.15 in the paper [43], which produces the, up to isomorphism and duality, 8 ordered sets of size 11 with the fixed point property and no retractable point. The second ordered set on Rutkowski's list, $P 2$, is shown in Fig. 2 . We have already seen $P 2$ "in action" with the counterexample $P 2^{a}$. On the positive side, $P 2$ also inspired the paper [42], which discusses the fixed point property for ordered sets that are built like $P 2$.

Beyond irreducible and retractable points, there are no convenient theorems to remove significant numbers of small sets from consideration. By generating the small ordered sets without a retractable point using Heitzig and Reinhold's algorithm (see [19]) and then checking the fixed point property with standard algorithms (see Sect. 9) for each one, the author found that, up to isomorphism and duality, there are 107 such sets of size 12, there are 946 such sets of size 13 and there are 24,546 such sets of size 14 . That is, the fixed point property experiences the "combinatorial explosion" that is quite typical for discrete problems.

\section{Products and Roddy's theorem (1994)}

Like the question for a characterization of the fixed point property, the question whether the product of two ordered sets with the fixed point property has the fixed point property, too, was asked by Rival, and it was first recorded in [5]. The product of a family of ordered sets is defined as follows. ${ }^{9}$

Definition 8.1 Let $\left\{P_{\alpha}\right\}_{\alpha \in I}$ be a family of ordered sets. Then the product $\prod_{\alpha \in I} P_{\alpha}$ is the set theoretical product of the sets $P_{\alpha}$ ordered by the pointwise order $\left(x_{\alpha}\right)_{\alpha \in I} \leq\left(y_{\alpha}\right)_{\alpha \in I}$ iff $x_{\alpha} \leq_{\alpha} y_{\alpha}$ for all $\alpha \in I$.

Along with the characterization question, this "product question" was a leading question in the fixed point theory of ordered sets. It inspired interesting proposed solutions (see, for example, [7,13]) as well as partial results in which fixed point theorems were translated into product theorems. Roddy's positive solution to the product question in the most important finite case was surprising in the sense that it started from scratch, without needing to refer to any previous work.

Theorem 8.2 (See [36], Theorem 1.1) Let $P$ and $X$ be finite ordered sets with the fixed point property. Then $P \times X$ has the fixed point property, too.

The proof, which we will only sketch, focuses not on the order-preserving function on the product $P \times X$ of two ordered sets, but on the factor maps that exist on the factors $P$ and $X$.

${ }^{9}$ We define arbitrary products, because we consider infinite products in Sect. 8.1. 
Definition 8.3 Let $P, X$ be ordered sets, let $f: P \times X \rightarrow P \times X$ be order-preserving and let $\Pi_{P}: P \times X \rightarrow P$ and $\Pi_{X}: P \times X \rightarrow X$ be the natural projections. For $x \in X$, we define the factor map $f_{x}: P \rightarrow P$ to be $f_{x}(\cdot):=\Pi_{P} f(\cdot, x)$. For $p \in P$, we define $f_{p}(\cdot):=\Pi_{X} f(p, \cdot)$.

Consider ordered sets $P$ and $X$ with the fixed point property. Note that, because the factor maps are orderpreserving, existence of a fixed point $p$ for a factor map $f_{x}$ is guaranteed. Similarly, it is guaranteed that there is a fixed point $x^{\prime}$ for the factor map $f_{p}$. Unfortunately it can be, and usually is, the case that $x^{\prime} \neq x$. Jumping back and forth between the ordered sets $P$ and $X$ in this fashion is unproductive, as, in each step, one hand destroys what the other hand has just repaired. The idea for an s-fence (see Definition 8.6), which is the key element of the proof, is to connect $p$ with a fixed point of $f_{x}$, where $x$ is so that $f_{p}(x)=x$. Before we consider s-fences, we note that, to avoid unnecessary iterations when a point is comparable to its image, the proof refers to flat maps and nice maps.

Definition 8.4 An order-preserving map $f: P \rightarrow P$ is called flat iff for all $x \in f[P]$ we have that $x$ is comparable to $f(x)$ iff $f(x)=x$. $f$ will be called a nice map iff

1. $f$ is flat,

2. $f$ is injective on its range,

3. There is a retraction $r_{f}: P \rightarrow f[P]$ such that $f=f \circ r_{f}$.

The following result shows that, for finite ordered sets, the proof can focus on functions whose factor maps on $P$ are nice.

Proposition 8.5 Let $P, X$ be finite ordered sets and let $g: P \times X \rightarrow P \times X$ be an order-preserving map. Then there is an order-preserving map $f: P \times X \rightarrow P \times X$ such that the following hold.

1. The factor maps $f_{x}: P \rightarrow P$ are all nice.

2. $x \leq y$ implies $r_{x}:=r_{f_{x}} \leq r_{f_{y}}=: r_{y}$.

3. $f$ has a fixed point iff $g$ has a fixed point.

The key to this result is that, on a finite set, sufficiently high powers of a function, such as $g^{n !}$ if the set has $n$ elements, are retractions. In a sense, this insight is similar to the Abian-Brown-style iteration, except that the starting points do not need to be comparable to their images. Concretely, the function $f: P \times X \rightarrow P \times X$ is defined by setting $f_{x}:=g_{x}^{|P| !+1}$ and $f_{p}:=g_{p}$ and all properties are readily verified.

The key definition for the proof is now that of an s-fence. Basically, if we have a fixed point $x \in X$ of a factor map $f_{a_{0}}: P \rightarrow P$, then we can consider the shortest distance from $a_{0}$ to a fixed point of $f_{x}$. It is not hard to show that this distance can be realized with an s-fence.

Definition 8.6 An $(n+2)$-tuple $\left(a_{0}, \ldots, a_{n} ; x\right)$ is called an up s-fence of length $n$ iff the following are satisfied.

1. $a_{0} \leq a_{1} \geq \cdots a_{n}$ in $P$,

2. $x \in X$,

3. $f_{a_{0}}(x)=x$,

4. $r_{x}\left(a_{j}\right)=a_{j}$, for $j>0$, and

5. $f_{x}\left(a_{n}\right)=a_{n}$.

A down s-fence is defined dually and an s-fence is one or the other.

Roddy's argument concludes by showing that the minimum length of an s-fence must be zero, which establishes the existence of a fixed point.

\subsection{Infinite ordered sets with the fixed point property}

After Roddy's Theorem settled the most important part of the product problem, namely, that the product of two finite ordered sets with the fixed point property has the fixed point property, too, for a while there was hope that there would be some ways to extend the argument to two arbitrary factors with the fixed point property. In fact, the first step is included in Roddy's argument: The fact that $X$ is finite, or indeed any property of $X$ except for the fixed point property, is not used in the proof. Hence the product of a finite ordered set with the fixed point property with any ordered set that has the fixed point property has the fixed point property, too. However, beyond this insight, a fully general proof that the fixed point property is "productive" has not materialized and neither have infinite counterexamples. Instead, the author has asked colleagues the following question. 
Open Question 8.7 (More of a meta-question) Are there any "truly" infinite ordered sets with the fixed point property?

This question requires some explanation, because, trivially, the natural numbers $\mathbb{N}$ with a top element $\infty$ attached are a chain-complete infinite ordered set with the fixed point property. However, $\mathbb{N} \cup\{\infty\}$ has the fixed point property for a rather trivial and finitary reason: It has a smallest element and the Abian-Brown Theorem does the rest. So, first of all, we dismiss from the discussion any infinite ordered sets that have the fixed point property for some trivial reason (smallest element, largest element, an element that is comparable to all other elements, a finite subset $A$ with the fixed point property so that every element outside $A$ is comparable to all elements of $A$, etc.).

As it turns out, all infinite ordered sets with the fixed point property that researchers are aware of have the fixed point property because of some combinatorial argument that ultimately forces the existence of a point $x \leq f(x)$. To the author, this means that such sets are not "truly" infinite with the fixed point property. Rather, they are "inflated finite sets."

To be sure, some arguments that show that a certain type of set is "close to being finite" require impressive machinery. For example, there is the non-trivial and very beautiful structure theorem by Li and Milner.

Theorem 8.8 (Li and Milner's Structure Theorem, see [27]) If $P$ is a chain-complete infinite ordered set with no infinite antichains, ${ }^{10}$ then there is a finite sequence $P=P_{0} \supseteq P_{1} \supseteq \cdots \supseteq P_{n}$ of subsets of $P$ so that there are retractions $r_{i}: P_{i-1} \rightarrow P_{i}$ with $r_{i}(p) \sim p$ for all elements of $P_{i-1}$ and so that $P_{n}$ is finite.

An argument similar to the proof of Theorem 4.4 shows that $P$ has the fixed point property iff $P_{n}$ does. Moreover, the argument can be extended to provide the most general product theorem for the fixed point property.

Theorem 8.9 (See Theorem 1.6 in [28]) The product of two ordered sets with the fixed point property so that at least one of them is chain-complete and has no infinite antichains has the fixed point property.

One can argue that, as long as, for all examples of infinite ordered sets with the fixed point property, the property is established with a combinatorial argument (such as dismantlability or the Abian-Brown Theorem) or an argument that involves finitely many elements (say, tracking where some special elements are mapped and using that the image set collapses into a finite or dismantlable set in each case), these ordered sets should be well-behaved when it comes to the formation of products. After all, if a combinatorial argument was enough to establish the fixed point property for the factors, there should be combinatorial arguments that do the same for products. (Basically, if we track finitely many points to establish the fixed point property, we could now track the same points in a similar way as components of elements of the product. The paper [41] is a good example of such a translation before Roddy's result was known.) Nonetheless, finitary or not, non-trivial results about infinite ordered sets with the fixed point property should remain interesting.

Coming back to the product problem, it is well-known that the corresponding problem in topology has a negative solution (see [6]): There are two topological spaces with the topological fixed point property (every continuous self-map has a fixed point) so that their product does not have the fixed point property. Before Roddy's Theorem, there was the suspicion that some triangulation of the spaces (there are examples that involve manifolds) could produce a counterexample that consists of simplicial complexes. Of course, for the finite case, this is now out of the question, but, maybe, there is a way to translate the topological example into infinite examples for the fixed point property for ordered sets. The main initial challenge here is that the topological examples are hard to visualize. Beyond that, the simplicial complexes obtained from infinite triangulations often fail to have the fixed point property in fairly obvious ways, or, the conditions that force the fixed point property are so strong that the fixed point property for products can be deduced from them, too. (And that, of course, feeds directly back into Open Question 8.7.)

Other attempts to work with infinite ordered sets with the fixed point property include Roddy's results in [37,38]. In [37], it is proved that, if $P$ and $Q$ have the fixed point property and $P$ has width 3 (no chain completeness demanded), then the product $P \times Q$ has the fixed point property. The argument is ingenious, but again combinatorial, as the proof establishes that the minimal elements of $P$ must have adjacent elements that form a special finite structure that forces fixed points in products.

The paper [38] considers the product $\prod_{j=1}^{\infty}\left(A_{2} \oplus F_{n}\right)$ of linear lexicographic sums of the antichain $A_{2}$ with 2 elements and fences $F_{n}$ with $n$ elements. All these sets are dismantlable, so there was hope that this set might have the fixed point property. Moreover, because the retractions used in the dismantlings of the factors

${ }^{10}$ An antichain is a set of elements so that no two distinct elements are comparable. 


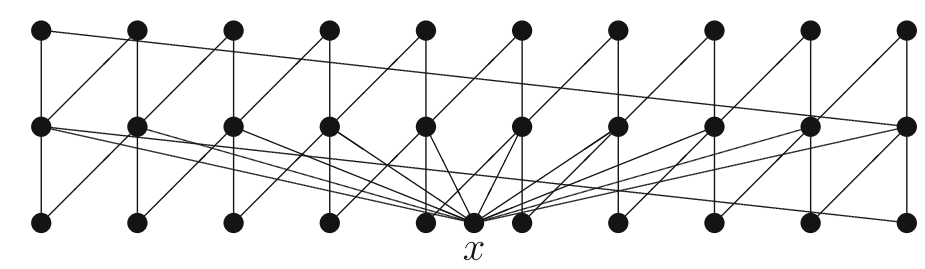

Fig. 6 An ordered set $P$ such that the power $\prod_{j=1}^{\infty} P$ has the fixed point property

cannot be translated into a dismantling of the whole product, there was hope that there would be an "infinitary" reason for the set to have the fixed point property. However, Roddy's argument in [38] shows that there is an infinitary reason why the set does not have the fixed point property: The construction of the fixed point free map requires the existence of a free ultrafilter on the natural numbers.

Existence of such a free ultrafilter can only be guaranteed by adding an axiom to the standard ZermeloFraenkel set theory axioms: At the least, we must demand that there is a free ultrafilter on the natural numbers. Of course, to assure that an infinite product is not empty, people typically assume some version of the Axiom of Choice (which implies, but is not implied by, the existence of free ultrafilters on the natural numbers; see [21] for more on the Axiom of Choice and its relatives). However, the sets $A_{2} \oplus F_{n}$ are all finite, and they are nested $A_{2} \oplus F_{n} \subseteq A_{2} \oplus F_{n+1}$. Therefore, we can assure that $\prod_{j=1}^{\infty}\left(A_{2} \oplus F_{n}\right)$ is not empty without even resorting to a mild version of the Axiom of Choice: Simply choose an element $x$ in the intersection $A_{2} \oplus F_{1}$, and $(x)_{n \in \mathbb{N}}$ is an element of the product. Hence there could be an interesting dichotomy if indeed the product $\prod_{j=1}^{\infty}\left(A_{2} \oplus F_{n}\right)$ could be proved to have the fixed point property when there are no free ultrafilters on the natural numbers.

Another possible source of infinite ordered sets with the fixed point property would be infinite powers of finite ordered sets, that is, infinite products of the same set.

Open Question 8.10 Are there two finite ordered sets $P$ and $Q$ so that $\prod_{j=1}^{\infty} P$ and $\prod_{j=1}^{\infty} Q$ each have the fixed point property, but so that the product $\prod_{j=1}^{\infty} P \times \prod_{j=1}^{\infty} Q=\prod_{j=1}^{\infty} P \times Q$ does not? Working towards this question, examples of ordered sets $P$ so that $\prod_{j=1}^{\infty} P$ has the fixed point property would be interesting in their own right. (Note that the question for a fixed point property for automorphisms was solved in [11].)

We focus on powers in Open Question 8.10, because infinite products of finite sets can fail to have the fixed point property for the very simple reason that they are disconnected. Consider the product $\prod_{j=1}^{\infty} F_{n}$. This is a product of dismantlable sets, because all factors are fences. However, the product is disconnected, because, with $l_{n}$ and $r_{n}$ denoting the leftmost and rightmost elements of $F_{n}$, respectively, the distance between the elements $\left(l_{n}\right)_{n=1}^{\infty}$ and $\left(r_{n}\right)_{n=1}^{\infty}$ is infinite, which means the product is disconnected.

Note that Open Question 8.10 at least provides the investigator a first target, namely to produce finite ordered sets whose infinite powers have the fixed point property. The author has produced a first family of such examples in [47]. It turns out that infinite powers of ordered sets as in Fig. 6 have the fixed point property as long as the bottom and top crowns have at least 10 elements each. The status of the fixed point property for the sets in this family that are constructed of 8-crowns or 6-crowns remains, strangely, unknown. The only thing that can be said for these cases is that the argument given in [47] (which is, of course, finitary and combinatorial) does not extend to them. Regarding the product problem, these sets are not much of a first step, as the products of these powers with chain-complete ordered sets with the fixed point property will have the fixed point property, too. True to form, the combinatorial argument that establishes the fixed point property for the power is so strong that it establishes the fixed point property for these products, too.

Then again, there may well be an approach to the product problem that does not require detailed knowledge of examples. The strong fixed point property of [13] guarantees that products have the fixed point property, but it was shown in [34] to not be equivalent to the fixed point property. The fixed point properties related to selection functions for certain set valued functions in [39] are similar in spirit to the strong fixed point property. It is currently unknown if the weakest of the properties in [39] is equivalent to the fixed point property or not. Such an equivalence, if it exists, would settle the product problem for chain-complete ordered sets without the need for a single example. 


\subsection{Nowakowski’s distortion}

There is an interesting generalization of the product question formulated by Nowakowski in 1981 which is not widely known. The author can only conjecture that this is because, before Roddy's Theorem, there was little hope to tackle harder problems, and, after Roddy's Theorem, there was a rush to declare the problems in this realm to be solved.

Definition 8.11 Let $P$ be an ordered set. Define the distortion $\operatorname{dist}(f)$ of an order-preserving function $f$ : $P \rightarrow P$ to be the smallest distance between a point $p$ and its image $f(p)$. Then define the Distortion $\operatorname{Dist}(P)$ of $P$ to be the maximum of all the distortions of order-preserving self-maps on $P$.

Open Question 8.12 Is it true that $\operatorname{Dist}(P \times Q)=\max \{\operatorname{Dist}(P), \operatorname{Dist}(Q)\}$ ?

In [33], Nowakowski conjectures that this is true. Clearly, Roddy's Theorem confirms the conjecture in case $P$ and $Q$ are finite and $\operatorname{Dist}(P)=\operatorname{Dist}(Q)=0$.

Examining Roddy's proof, it can be seen that Proposition 8.5 works for this situation, too, with the third part replaced with $\operatorname{dist}(f)=\operatorname{dist}(g): \operatorname{dist}(f) \leq \operatorname{dist}(g)$ follows from the fact that the factor maps $f_{x}$ are iterations of $g_{x}$ and $f_{p}=g_{p} ; \operatorname{dist}(f) \geq \operatorname{dist}(g)$ follows from the fact that the distortion will be realized for a point $(p, x)$ with $(p, x)=r(p, x)$.

Regarding the s-fence technique, if it works for this problem, the translation is not completely obvious. The author tried to generalize Roddy's argument for the next $\operatorname{simplest}$ case $\operatorname{Dist}(P)=0$ and $\operatorname{Dist}(X)>0$, only to realize that, in the iterative process that happens in $P$, the distortions of the factor maps on $X$ are not easily guaranteed to stay minimal, even if an explicit example eluded the author. Similarly, it is not obvious how to generalize the technique for the other possibilitity $(\operatorname{Dist}(P)>0$ and $\operatorname{Dist}(X)=0)$.

\section{Duffus and Goddard's NP-completeness proof and algorithmic approaches (1996)}

As we have seen, beyond successes for special classes, the characterization of the fixed point property for finite sets in general looked like a daunting task. In 1996, Duffus and Goddard's paper [12] showed why this is so: The decision problem

Given. A finite ordered set $P$.

Question. Does $P$ have a fixed point free order-preserving self-map?

is NP-complete. Although the constructions differ, this result was in some ways foreshadowed by [52] in which Williamson, a student of Duffus, proved that it was NP-complete to decide if a finite ordered set had a fixed point free automorphism. Both proofs show that it is possible to construct ordered sets based on instances of 3SAT so that the ordered set has a fixed point free self-map (or automorphism) iff the given instance of 3SAT is satisfiable.

Although NP-completeness means that a problem must be considered hard, barring a solution to the $\mathrm{P}$ versus NP problem, it is interesting to determine special cases that are solvable in polynomial time. For example, there is a polynomial algorithm that determines if an ordered set is dismantlable by irreducibles: Check if the set has an irreducible point, remove the first irreducible point you find, continue this process with the new smaller set until no more irreducible points are found. $P$ is dismantlable by irreducibles iff this process ends with a singleton ordered set. Hence we can determine in polynomial time whether an ordered set of height 1 or width 2 has the fixed point property (see Theorem 4.12 and the remarks thereafter). On the other hand, the process to check if an ordered set of (interval) dimension 2 has the fixed point property (see remarks after Theorem 4.15) is only guaranteed to terminate in polynomial time if we require bounded height. This leads to a number of open questions.

Open Question 9.1 For what classes of ordered sets can it be decided in polynomial time if a set $P$ in the class has the fixed point property? Width 3? (Author conjecture: Yes.) Height 2? (Author conjecture: No, unless $P=N P$.) (Interval) dimension 2? (Author conjecture: Yes.)

In addition to direct approaches using theorems in order theory, the constraint satisfaction problem framework from computer science (see [50]) can be adapted to finding a fixed point free order-preserving self-map of a finite ordered set $P$ as follows.

Definition 9.2 Let $P$ be a finite ordered set. For all $x, y \in P$ with $x \nsim y$, let $(x, y)$ be a vertex in a graph $G$. Two vertices $\left(x_{1}, y_{1}\right)$ and $\left(x_{2}, y_{2}\right)$ are joined by an edge iff $x_{1} \neq x_{2}$ and both of the logical propositions 
$x_{1}<x_{2} \Rightarrow y_{1} \leq y_{2}$ and $x_{2}<x_{1} \Rightarrow y_{2} \leq y_{1}$ are true. Consistent with the language in constraint satisfaction (see, for example, [31]), which applies in much more general settings, we call this graph the expanded constraint network for the fixed point problem.

Cliques in the expanded constraint network for the fixed point problem are fixed point free order-preserving partial self-maps of $P$ and $P$ has a fixed point free order-preserving self-map iff there is a clique of size $|P|$ in the expanded constraint network.

Through this connection, on one hand, search algorithms, such as discussed in [26], can be applied to determine if there is a fixed point free order-preserving self-map. Readers who wish to try out the Constraint Satisfaction Problem Solver at [48] will find most of these algorithms to be very effective for the fixed point property. On the other hand, many of the known polynomial preprocessors for these search algorithms are promising for the fixed point property: For any ordered set with up to 100 elements that the author tried with [48], the fixed point property was rather easy to decide.

One of these preprocessors, called path consistency, was rediscovered in the context of the fixed point property for ordered sets in [53]. (In artificial intelligence, it was already known in [29].) To enforce path consistency, for every edge $\left\{\left(x_{1}, y_{1}\right),\left(x_{2}, y_{2}\right)\right\}$ in the expanded constraint network and for every $x_{3} \notin\left\{x_{1}, x_{2}\right\}$, check if there is a $y_{3}$ so that $\left(x_{3}, y_{3}\right)$ is a vertex and so that both $\left\{\left(x_{1}, y_{1}\right),\left(x_{3}, y_{3}\right)\right\}$ and $\left\{\left(x_{3}, y_{3}\right),\left(x_{2}, y_{2}\right)\right\}$ are edges in the expanded constraint network. If this is not the case, then $\left\{\left(x_{1}, y_{1}\right),\left(x_{2}, y_{2}\right)\right\}$ cannot be an edge in a clique of size $|P|$ and hence it can be removed. This process continues until no further edges can be removed and it terminates in polynomial time. If the process ends with an empty expanded constraint network, then there is no clique of size $|P|$ and hence $P$ has the fixed point property. If the process ends with a non-empty expanded constraint network, we must still use a search algorithm to determine if there is a fixed point free order-preserving self-map.

Xia proved in [53] that, if $P$ is dismantlable by irreducibles, then enforcing path consistency on the expanded constraint network for the fixed point problem produces an empty graph. This should not be surprising, because dismantlability by irreducibles can be checked in polynomial time. In [10], a similar theorem is proved for the ordered sets discussed in [14]. It seems that enforcing path consistency is a much stronger sufficient criterion for checking the fixed point property than indicated by the results that can currently be proved. To the author's knowledge, the smallest ordered set with the fixed point property for which enforcing path consistency returns a non-empty expanded constraint network has more than 400 points and it is constructed as in [12], using the smallest non-satisfiable instance of 3SAT (all 8 clauses on 3 variables). We are led to a wide open question.

Open Question 9.3 For which ordered sets with the fixed point property will the enforcing of path consistency return an empty expanded constraint network?

Partial results, such as showing that this is the case for width 3 or (interval) dimension 2 (the author conjectures this is true) or for height 2 (the author conjectures this is false) would be interesting, too. An interesting borderline case would be the collapsible ordered sets from the end of Sect. 4. Their recursive structure is worst-case exponential, but (see [45]) polynomial if we demand a uniform bound on the height. Moreover, for collapsible ordered sets of height 2 with the fixed point property, enforcing path consistency will return an empty constraint network. It is unknown is this remains true when we drop the requirement that the height be 2 .

\section{Starting back up from scratch? Fixed vertices in graphs (2012)}

In graph theory, homomorphisms are functions on the vertices that preserve adjacencies (see [20]).

Definition 10.1 If $G=(V, E)$ and $H=(W, F)$ are graphs, then $f: V \rightarrow W$ is a homomorphism iff $\left\{v_{1}, v_{2}\right\} \in E$ implies that $\left\{w_{1}, w_{2}\right\} \in F$.

Because graphs typically do not have loops at their vertices, the order-theoretical analogue of homomorphisms are the strictly order-preserving functions, which are not as widely analyzed as order-preserving functions. If we require loops at the vertices, the remarks before Definition 6.3 indicate that a fixed point property for graphs with loops is not very interesting. However, this changes drastically for graphs without loops.

Definition 10.2 A graph $G$ without loops has the fixed vertex property iff, for every homomorphism $f$ from $G$ to itself, there is a vertex $v$ so that $f(v)=v$. 
By replacing the directed edges of a directed graph with what graph theorists call a "gadget" in such a way that endomorphisms must map gadgets isomorphically to gadgets, we can obtain an undirected graph so that there is a one-to-one correspondence between the endomorphisms of the directed graph and the endomorphisms of the new undirected graph. Recently, the author has found gadgets that can be used to represent directed edges and loops and which work so that their products act like gadgets in products. Therefore the whole fixed point theory for ordered sets can be embedded into a completely unexplored fixed vertex theory for graphs without lops.

Because it is NP-complete to decide if a given ordered set has a fixed point free order-preserving self-map, it is NP-complete to decide if a given graph has a fixed vertex-free endomorphism. However, the fixed vertex property will require a whole new set of tools to investigate. Although Theorem 4.2 has an analogue for the fixed vertex property, there is no Abian-Brown Theorem (a cycle is a natural counterexample), neither direction of Theorem 4.4 holds, and there are lexicographic sums so that the index graph as well as all the pieces have the fixed vertex property, but the overall graph does not.

On the positive side, because the fixed vertex property requires fresh ideas, graph theoretical proofs could shed a new light on fixed point results in ordered sets. It is not known if the product of two connected graphs with the fixed vertex property must have the fixed vertex property. Because of the absence of an Abian-Brown Theorem for graphs, it may well be that a proof for this result (or a counterexample) points the way towards a full resolution of the product question for the fixed point property.

\section{Conclusion (The Future)}

We have gained an overview of the substantial body of combinatorial results for the fixed point property. With online searches giving the latest references quickly and easily, the bibliography is focused only on the works cited in the text. More references, results and open problems can be found in [46].

The open questions as well as the remarks given here show that several established parts of the theory, such as the connection to algebraic topology and the product question merit further investigation, possibly from a different viewpoint, such as fixed simplices in simplicial complexes or fixed vertices in graphs. Moreover, connections to new fields, such as fixed vertices in graphs, consistency algorithms in constraint satisfaction, or discrete Morse theory, are rather new and wide open for exploration. To this author, discrete concepts for which the Abian-Brown Theorem fails (see Sects. 6, 10) are particularly interesting, as arguments in these situations may also shed a light on infinite ordered sets with the fixed point property.

It will be interesting to see where the journey leads next.

Open Access This article is distributed under the terms of the Creative Commons Attribution License which permits any use, distribution, and reproduction in any medium, provided the original author(s) and the source are credited.

\section{References}

1. Anonymous referee.: Private communication

2. Abian, A.: Fixed point theorems of the mappings of partially ordered sets. Rendiconti del Circolo Mathematico di Palermo 20, 139-142 (1971)

3. Abian, S.; Brown, A.B.: A theorem on partially ordered sets with applications to fixed point theorems. Can. J. Math. 13, 78-82 (1961)

4. Baclawski, K.: A combinatorial proof of a fixed point property. J. Combin. Theory (A) 119, 994-1013 (2012)

5. Baclawski, K.; Björner, A.: Fixed points in partially ordered sets. Adv. Math. 31, 263-287 (1979)

6. Brown, R.: The fixed point property and cartesian products. Am. Math. Mon. 89, 654-678 (1982)

7. Corominas, E.: Sur les ensembles ordonnés projectifs et la propriété du point fixe. C. R. Acad. Sci. Paris 311(Série 1), 199-204 (1990)

8. Davey, B.; Priestley, H.: Introduction to Lattices and Order. Cambridge University Press, Cambridge (1990)

9. Davis, A.C.: A characterization of complete lattices. Pac. J. Math. 5, 311-319 (1955)

10. Donalies, M.; Schröder, B.: Performance guarantees and applications for Xia's algorithm. Discrete Math. 213, 67-86 (2000). In: Proceedings of the Banach Center Minisemester on Discrete Mathematics, week on Ordered Sets

11. Duffus, D.: Automorphisms and products of ordered sets. Algebra Univ. 19, 366-369 (1984)

12. Duffus, D.; Goddard, T.: The complexity of the fixed point property. Order 13, 209-218 (1996)

13. Duffus, D.; Sauer, N.: Fixed points of products and the strong fixed point property. Order 4, 221-231 (1987)

14. Edelman, P.: On a fixed point theorem for partially ordered sets. Discrete Math. 15, 117-119 (1979)

15. Fofanova, T.; Rutkowski, A.: The fixed point property in ordered sets of width two. Order 4, 101-106 (1987) 
16. Fofanova, T.; Rival, I.; Rutkowski, A.: Dimension 2, fixed points and dismantlable ordered sets. Order 13, 245-253 (1994)

17. Heikkilä, S.: On fixed points through a generalized iteration method with applications to differential and integral equations involving discontinuities. Nonlinear Anal. 14, 413-426 (1990)

18. Heikkilä, S.; Lakhshmikantham, V.: Monotone Iterative Techniques for Discontinuous Nonlinear Differential Equations. Marcel Dekker, New York (1994)

19. Heitzig, J.; Reinhold, J.: The number of unlabeled orders on fourteen elements. Order 17, 333-341 (2000)

20. Hell, P.; Nešetril, J.: Graphs and Homomorphisms. Oxford Lecture Series in Mathematics and its Applications, vol. 28. Oxford University Press, Oxford (2004)

21. Herrlich, H.: Axiom of Choice. Springer, Berlin (2006)

22. Höft, H.; Höft, M.: Some fixed point theorems for partially ordered set. Can. J. Math. 28, 992-997 (1976)

23. Höft, H.; Höft, M.: Fixed point invariant reductions and a characterization theorem for lexicographic sums. Houston J. Math. 14(3), 411-422 (1988)

24. Höft, H.; Höft, M.: Fixed point free components in lexicographic sums with the fixed point property. Demonstratio Math. XXIV, 294-304 (1991)

25. Knaster, B.: Un théorème sur les fonctions d'ensembles. Ann. Soc. Polon. Math. 6, 133-134 (1928)

26. Kondrak, G.; van Beek, P.: A theoretical evaluation of selected backtracking algorithms. Artif. Intell. 89, 365-387 (1997)

27. Li, B.; Milner, E.C.: A chain complete poset with no infinite antichain has a finite core. Order 10, 55-63 (1993)

28. Li, B.; Milner, E.C.: From finite posets to chain complete posets having no infinite antichain. Order 12, 159-171 (1995)

29. Mackworth, A.K.: Consistency in networks of relations. Artif. Intell. 8, 99-118 (1977)

30. Maróti, M.; Zádori, L.: Reflexive digraphs with near unanimity polymorphisms. Discrete Math. 312, 2316-2328 (2012)

31. Nadel, B.: Constraint satisfaction algorithms. Comput. Intell. 5, 188-224 (1989)

32. Nowakowski, R.; Rival, I.: A fixed edge theorem for graphs with loops. J. Graph Theory 3, 339-350 (1979)

33. Nowakowski, R.: Open question on p. 842 of I. Rival, ed. (1982), Ordered sets. Reidel, Boston (1981)

34. Pickering, D.; Roddy, M.: On the strong fixed point property. Order 9, 305-310 (1992)

35. Rival, I.: A fixed point theorem for finite partially ordered sets. J. Combin. Theory (A) 21, 309-318 (1976)

36. Roddy, M.: Fixed points and products. Order 11, 11-14 (1994)

37. Roddy, M.: Fixed points and products: width 3. Order 19, 319-326 (2002)

38. Roddy, M.: On an example of Rutkowski and Schröder. Order 19, 365-366 (2002)

39. Roddy, M.; Schröder, B.: Isotone relations revisited. Discrete Math. 290, 229-248 (2005)

40. Rutkowski, A.: The fixed point property for small sets. Order 6, 1-14 (1989)

41. Rutkowski, A.; Schröder, B.: Retractability and the fixed point property for products. Order 11, 353-359 (1994)

42. Rutkowski, A.; Schröder, B.: A fixed point theorem with applications to truncated lattices. Algebra Univ. 53, 175-187 (2005)

43. Schröder, B.: Fixed point property for 11-element sets. Order 10, 329-347 (1993)

44. Schröder, B.: Algorithms for the fixed point property. Theor. Comput. Sci. 217, 301-358 (1996)

45. Schröder B.: Fixed cliques and generalizations of dismantlability. In: Alavi, Y.; et al.: Combinatorics, Graph Theory, and Algorithms. Proceedings of the Eighth Quadrennial International Conference in Graph Theory, Combinatorics, Algorithms and Applications, vol. II, pp. 747-756 (1996)

46. Schröder, B.: Ordered Sets: An Introduction. Birkhäuser Verlag, Boston (2003)

47. Schröder, B.: Examples of powers of ordered sets with the fixed point property. Order 23, 211-219 (2006)

48. http://www2.latech.edu/schroder/software.htm

49. Tarski, A.: A lattice-theoretical fixpoint theorem and its applications. Pac. J. Math. 5, 285-309 (1955)

50. Tsang, E.: Foundations of Constraint Satisfaction. Academic Press, New York (1993)

51. Willard, S.: General Topology. Addison-Wesley, Reading (1970)

52. Williamson, S.: Fixed point properties in ordered sets. Ph.D. dissertation, Emory University (1992)

53. Xia, W.: Fixed point property and formal concept analysis. Order 9, 255-264 (1992) 